\title{
The Isotopx NGX and ATONA Faraday amplifiers
}

\author{
Stephen E. Cox ${ }^{1}$, Sidney R. Hemming ${ }^{1,2}$, and Damian Tootell ${ }^{3}$ \\ ${ }^{1}$ Lamont-Doherty Earth Observatory, the Earth Institute at Columbia University, 61 Route 9W, Palisades, NY 10964, USA \\ ${ }^{2}$ Department of Earth and Environmental Sciences, Columbia University, New York, NY 10027, USA \\ ${ }^{3}$ Isotopx, Ltd., Dalton House, Dalton Way, Middlewich CW10 0HU, UK
}

Correspondence: Stephen E. Cox (stephen@stephencoxgeology.com)

Received: 22 January 2020 - Discussion started: 7 February 2020

Revised: 10 May 2020 - Accepted: 12 May 2020 - Published: 3 September 2020

\begin{abstract}
We installed the new Isotopx ATONA Faraday cup detector amplifiers on an Isotopx NGX mass spectrometer at Lamont-Doherty Earth Observatory in early 2018. The ATONA is a capacitive transimpedance amplifier, which differs from the traditional resistive transimpedance amplifier used on most Faraday detectors for mass spectrometry. Instead of a high-gain resistor, a capacitor is used to accumulate and measure charge. The advantages of this architecture are a very low noise floor, rapid response time, stable baselines, and very high dynamic range. We show baseline noise measurements and measurements of argon from air and cocktail gas standards to demonstrate the capabilities of these amplifiers. The ATONA exhibits a noise floor better than a traditional $10^{13} \Omega$ amplifier in normal noble gas mass spectrometer usage, superior gain and baseline stability, and an unrivaled dynamic range that makes it practical to measure beams ranging in size from below $10^{-16}$ to above $10^{-9} \mathrm{~A}$ using a single amplifier.
\end{abstract}

\section{Introduction}

The design of analog ion collectors for mass spectrometry has changed strikingly little for 70 years. Early instruments already employed much of the detector technology we recognize today, including multiple collectors, secondary electron suppressors, and electronic circuits that employed highvalue resistors (resistor transimpedance amplifiers, or RTIA) to amplify small currents to measurable voltages (e.g., Nier, 1940, 1947). Between the 1950s and 1980s, as the field of isotope geochemistry shifted from home-brewed instruments to commercial ones, available noble gas mass spectrometers consolidated around a design based on the Reynolds mass spectrometer using a "Nier-type" ion source, a fixed accelerating voltage, a variable magnetic field, and a single pair of collectors consisting of an analog electron multiplier (later an ion-counting multiplier) and a Faraday cup, intended to be used separately for signals of different sizes (e.g., Reynolds, 1956; Bayer et al., 1989; Renne et al., 1998; Burnard and Farley, 2000). Since around 2010, multicollection has come back into vogue as improvements in electronic noise and stability have mitigated the problems of comparing beams measured on two separate amplifiers, and the field has sought ways to minimize the uncertainty conferred by the fitting of gas evolution trends in order to calculate isotope ratios at the time of sample inlet (e.g., Mark et al., 2009; Coble et al., 2011).

The shift toward multicollection has been accompanied by a diversification of the collector technologies available, with new ion-counting multipliers built with a geometry that allows multicollector spacing and new RTIA Faraday amplifiers employing higher-value resistors in order to take advantage of the $\sqrt{R}$ relationship between normalized signal noise and resistance (e.g., Zhang et al., 2016). These advances are not without trade-offs, however. For one, multicollection requires the use of wider flight tubes and larger collector blocks that increase the volume of static vacuum instruments, reducing their effective sensitivity; some applications may still benefit from the use of small-volume single-collector instruments, for which fast, high-dynamic-range detectors are particularly valuable. On some multicollector instruments, the use of ion-counting multipliers in the detector position for large beams $\left({ }^{40} \mathrm{Ar}\right.$, for example) allows the measurement of very small samples but limits the dynamic range (Jicha et al., 2016). Instruments using high-value resistor amplifiers to achieve the same goal also suffer from a loss of dynamic 
range, although it is not as severe, but additionally suffer from long settling times (large tau), baseline instability, and drift in gain calibration. The settling time problem is less severe on multicollectors that do not need to peak hop but can still affect signal stability during the start of a measurement. These problems have limited the use of such collectors for decades, but the cost-benefit calculation has shifted due to improving electronic stability and new techniques for dealing with the tau correction (Zhang et al., 2016), as well as a cultural shift in the priorities of noble gas geochemistry labs toward young, small samples and higher precision (e.g., Wijbrans et al., 2011; Jicha et al., 2012; Mark et al., 2017; Rose and Koppers, 2019).

However, the desire to measure young samples well has not displaced the need to measure old samples very precisely (Sprain et al., 2015), to measure large amounts of noble gas in ice core and water samples (Lu et al., 2014), and to measure extreme abundance ratios such as those that are typical in ${ }^{3} \mathrm{He} /{ }^{4} \mathrm{He}$ analyses (Espanon et al., 2014). The ideal collector, therefore, has not just a low noise floor and high sensitivity, but also a high dynamic range, the ability to switch between low and high signals with no memory, and a stable, precisely measurable gain bias between each detector. Resistor Faraday amplifiers have a fairly restricted dynamic range, with the ability to reliably measure signals over only about 5 orders of magnitude. Ion-counting multipliers are only able to measure small signals and suffer from significant nonlinearity at the upper and lower ends of their useful range. Analog multipliers have a much higher dynamic range, about 8 orders of magnitude, but suffer from both nonlinearity and relatively short-timescale gain drift. In addition, electron multipliers wear quickly and are both expensive and vulnerable to damage from vacuum accidents and large ion beams. Faraday cups are extremely linear, quiet, resilient, and cheap to manufacture, so a technological solution that extends their useful dynamic range and sensitivity to small signals is highly desirable.

Mass spectrometers have always relied on transimpedance amplifiers, which consist of an active circuit element (usually an op-amp) that converts a small input current to a high output voltage (Fig. 1). The capacitive transimpedance amplifier was developed decades ago and was an option on such venerable devices as the Keithley 6512 Electrometer, which provided the option of feedback resistors or capacitors for current measurements. The advantage of the latter was seen as the high dynamic range, while the disadvantages were the accuracy and linearity. More recent work has demonstrated the promise of low noise and stability using feedback capacitors but with serious limitations on dynamic range, linearity, and flexibility caused by the measurement of accumulated charge and the need to handle routine discharging of the feedback capacitor (Esat, 1995; Ireland et al., 2014). The new ATONA capacitive transimpedance amplifier developed by Isotopx maintains the high dynamic range (effectively unlimited for noble gas measurements) and rapid response time

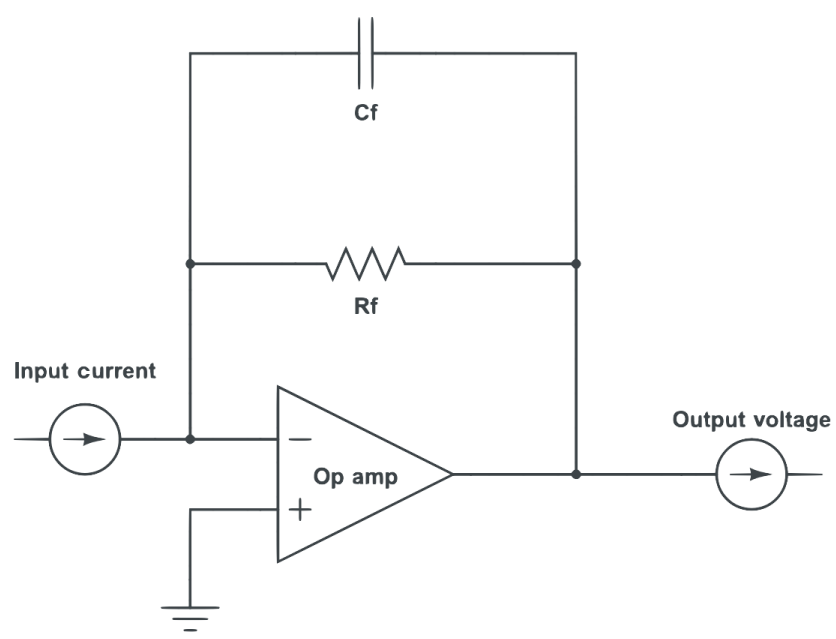

Figure 1. Schematic of a transimpedance amplifier. Note that practical examples are far more complex. The circuit consists of an opamp, which is the active element that converts the input current to a proportional output voltage, and then a feedback resistor and capacitor that determine the gain of the circuit. In a traditional resistance transimpedance amplifier, the resistor is very high value and the capacitance is reduced as much as is practical. The ATONA instead uses a defined capacitance as the feedback element.

of the earlier feedback capacitor devices while also delivering the linearity and accuracy more traditionally associated with resistor transimpedance amplifiers. The ATONA uses a proprietary extremely low-leakage dielectric for the feedback capacitor combined with a cooled amplifier housing to reduce the leakage current, and consequent nonlinearity, to below $1 \mathrm{ppm}$. Unlike previous charge-mode amplifiers, the ATONA measures the rate of change of the transimpedance amplifier output voltage and therefore the rate of change of the accumulated charge. The advantages of this setup, which can accurately measure extremely low signals without sacrificing stability or the ability to measure large signals, are significant for noble gas mass spectrometry and for mass spectrometry in general.

Noble gas mass spectrometers must measure an evolving signal due to the action of the instrument itself on the sample (Fig. 2). Sample abundances are typically so small that the entire sample is allowed to equilibrate with the vacuum inside the mass spectrometer at the beginning of analysis, which requires that the pumps be isolated from the vacuum chamber. Starting at this time, confounding gases will be introduced through undetectable leaks and desorption from the walls of the vacuum chamber housing the mass spectrometer, and sample gas will be consumed by ionization in the ion source and implantation in either the collector or the walls of the vacuum chamber. Because these processes change the gas composition, and therefore both the abundances and the ratios of the noble gas isotopes being measured, noble gas geochemists typically extrapolate the evolving gas signal back 


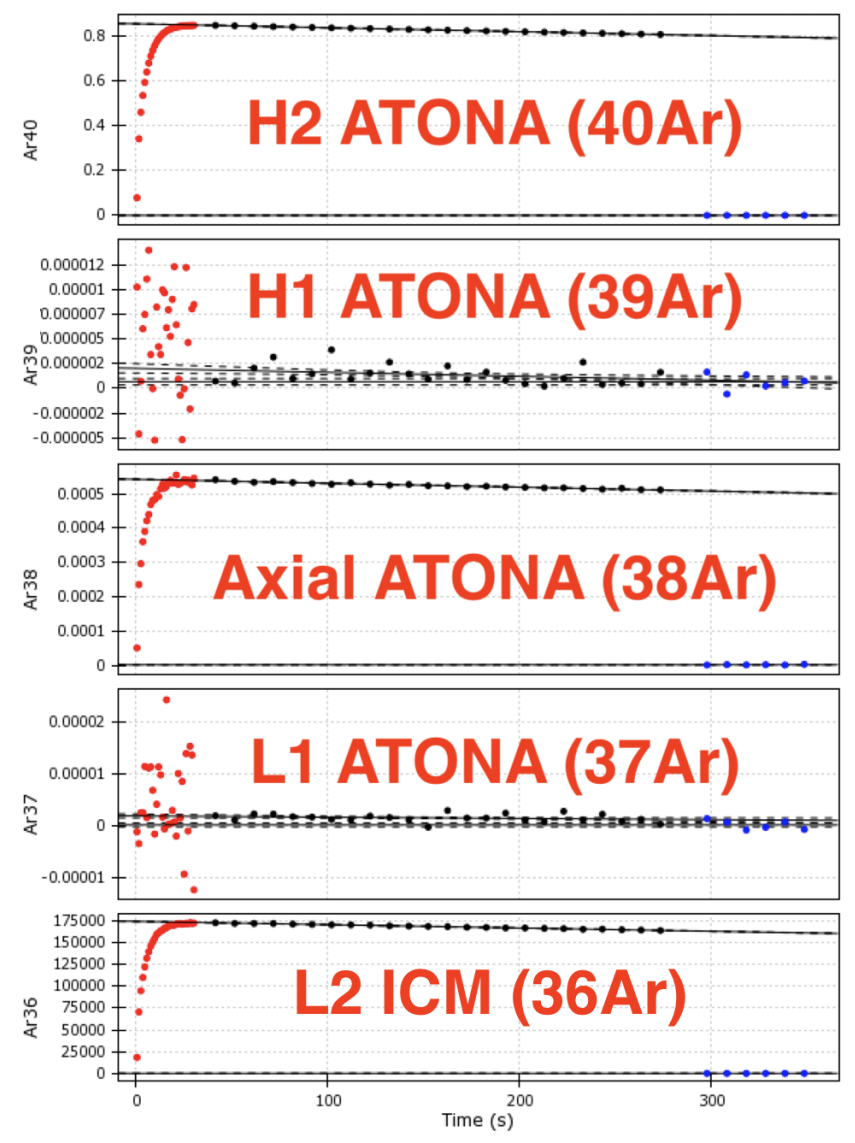

Figure 2. An example measurement of the $8.5 \times 10^{-13} \mathrm{~mol}^{40} \mathrm{Ar}$ air standard on the Isotopx NGX. The gas is measured with a $1 \mathrm{~s}$ integration time during $30 \mathrm{~s}$ of sample inlet, then with a $10 \mathrm{~s}$ integration time during $240 \mathrm{~s}$ of measurement and $60 \mathrm{~s}$ of baseline measurement. The figure shows the live measurement screen displayed in Pychron during automated sample analysis with overlain labels. Each signal is displayed as reported by the Isotopx software: volts for the four Faraday collectors (converted by the onboard ATONA firmware to equivalent $10^{11} \Omega$ RTIA volts) and counts per second for the ion-counting electron multiplier.

to the time of sample inlet - commonly referred to as "time zero" - meaning that the analysis loses statistical power as it continues in time. The advent of multicollection means that isotope ratios could be computed directly at each time point and then themselves extrapolated to time zero, but so far noble gas geochemists have largely used multicollection simply as a means to ensure that the maximum amount of data can be collected simultaneously for each isotope.

\section{Isotopx NGX and ATONA amplifier}

The Isotopx NGX is a multicollector noble gas mass spectrometer with a Nier-type ion source, a Hall probe feedback-controlled electromagnet mass analyzer, and a customizable collector block comprising fixed Faraday cup and ion-counting electron multiplier detectors. The source sensitivity is approximately $10^{-3} \mathrm{~A}$ per Torr, the ${ }^{36} \mathrm{Ar}$ background is approximately $2 \times 10^{-19} \mathrm{~mol}$, or $5 \times$ $10^{-15} \mathrm{cc} \mathrm{STP}$, and the rise is approximately $8 \times 10^{-18} \mathrm{~mol}$, or $2 \times 10^{-13} \operatorname{cc~STP}^{40} \mathrm{Ar} \mathrm{min}^{-1}$. The NGX at the LamontDoherty Earth Observatory (LDEO) has five fixed detectors, four Faraday cups, and one electron multiplier in the appropriate configuration to simultaneously collect the five isotopes of argon typically measured for ${ }^{40} \mathrm{Ar} /{ }^{39} \mathrm{Ar}$ dating: ${ }^{40} \mathrm{Ar},{ }^{39} \mathrm{Ar},{ }^{38} \mathrm{Ar},{ }^{37} \mathrm{Ar}$, and ${ }^{36} \mathrm{Ar}$. The electron multiplier is placed at the ${ }^{36} \mathrm{Ar}$ position, where signals are typically relatively small and must be measured with high precision due to the need for an accurate ${ }^{40} \mathrm{Ar} /{ }^{36} \mathrm{Ar}$ ratio for initial $\mathrm{Ar}$ correction. We chose this configuration before the ATONA became available, and in fact we believe that an ATONA would be appropriate for ${ }^{36} \mathrm{Ar}$ measurement in many situations. An instrument with the ability to switch between measuring ${ }^{36} \mathrm{Ar}$ on an ATONA and an electron multiplier would be able to take advantage of the stability and dynamic range of the ATONA for large ${ }^{36} \mathrm{Ar}$ signals while still using an ioncounting electron multiplier for very small signals. For example, a single heating step on a very young basalt sample may yield $10^{-14} \mathrm{~mol}$ of ${ }^{40} \mathrm{Ar}, 95 \%$ of which is non-radiogenic. In this case, the uncertainty of the ${ }^{36} \mathrm{Ar}$ measurement will dominate the trapped $\mathrm{Ar}$ correction to the ${ }^{40} \mathrm{Ar}$ and therefore the age uncertainty, and we would choose to measure the $3 \times 10^{-17} \mathrm{~mol}^{36} \mathrm{Ar}$ signal with the ion counter with $0.2 \%$ uncertainty rather than using the ATONA with $3 \%$ uncertainty.

After initial installation in late 2017 with Isotopx $10^{11} \Omega$ and $10^{12} \Omega$ Xact amplifiers, we installed a prototype set of ATONA amplifiers on the NGX in March 2018. The ATONA is a capacitive transimpedance amplifier, which is partially described in UK patent GB2552232 (https://www.ipo.gov. uk/p-ipsum/Case/PublicationNumber/GB2552232, last access: 21 August 2020). The remaining aspects of the amplifier are protected as trade secrets. The ATONA substitutes the typical high-gain resistor of an RTIA, for which one would try to minimize the capacitance of the circuit, with a capacitor and a series of proprietary circuits that allow the rate of charge accumulation (rather than the accumulated charge itself) to be continuously sampled (again, the exact mechanism used is a trade secret). Because the ATONA relies on a measurement of the rate of charge accumulation, it simply discharges the feedback capacitor when the rated capacitance has been reached in a process that is transparent to the measurement itself. The proprietary paraelectric dielectric material minimizes nonlinearity due to current leakage and dielectric hysteresis. Because the Faraday buckets are directly connected to the input of the inverting amplifier, the voltage of the bucket is fixed at zero volts regardless of the accumulated charge on the capacitor, and therefore charge buildup that might affect ion behavior is avoided. The result is that the ATONA can measure a wide range of ion beam currents, from attoamps to nanoamps (hence the name), with good linearity, very low noise, and a settling time short enough to be 
insignificant (less than the $2 \mathrm{~ms}$ sampling time of the measurement electronics).

The ATONA has the important characteristic that the noise scales inversely with time, rather than with the square root of time, so accumulating a signal for longer between sampling intervals will result in a linearly less noisy signal. Counting statistics reduces uncertainty with the square root of time, so by comparison the ATONA gains an additional factor of the square root of time in noise reduction when the sampling interval is extended. There is a trade-off in noble gas mass spectrometry because of the evolution of the signal with time, although it is important to mention that the signal from the production version of the ATONA can be subsampled without sacrificing the gain of the longer sampling time. This dynamic opens up a wide array of possibilities of best measurement practice that will vary with ion beam size, and we have not yet fully explored them; for example, one might choose a longer integration time for smaller beams that are measured as an average and a shorter integration time for larger beams during the same measurement. The work presented here has led us to settle on an integration time of $10 \mathrm{~s}$, with a typical total analysis time of $600 \mathrm{~s}$ in multicollection mode, as a sweet spot for reducing noise without sacrificing gas evolution fit statistics. Analytical conditions for different experiments in this study vary and are described in the figure captions. All isotope evolutions are fit using a linear regression with no outlier data points excluded from either fits or uncertainty calculations and with no measurement cycles discarded from the analysis. The only exception is in Sect. 3.3, in which we removed the final $200 \mathrm{~s}$ from a set of $600 \mathrm{~s} \mathrm{Ar-}$ gon Intercalibration Pipette System (APIS) analyses in order to allow a direct comparison to a dataset of $400 \mathrm{~s}$ analyses on a different mass spectrometer.

\section{Analyses of electronics and gas standards}

\subsection{Background noise}

Reported detector signal units are an arbitrary choice in mass spectrometry; the important quantity for a given detector is the signal-to-noise ratio produced by a given incident ion beam. We quantify this by converting measured signal from detector units to incident ion beam current using Ohm's law for voltage measured on an RTIA. The ATONA does not measure voltage in the same way as an RTIA, but its firmware converts the signal to equivalent $10^{11} \Omega$ RTIA volts. We convert back to beam current for clearer comparison with RTIAs that have a different gain and with other types of detectors. As an example, $1 \times 10^{11} \Omega$ RTIA volts is equivalent to $10^{4} \mathrm{fA}$, and $625 \mathrm{cps}$ on an ion-counting electron multiplier is equivalent to $0.1 \mathrm{fA}$. We calculate background noise for ideal RTIAs with a variety of feedback resistors. In this case, we assume that the only significant component of noise is Johnson-Nyquist (J-N) noise, or thermal white noise, which is an inherent property of all conductors. The observed noise is caused by the movement of charge within the conductor in response to random fluctuations caused by thermal radiation, as described by Nyquist (1928) (see Appendix A for the equation). $\mathrm{J}-\mathrm{N}$ noise provides an absolute limit for the signal-to-noise ratio achievable with an RTIA, and the best commercial RTIAs approach this limit.

Unlike J-N noise, kTC noise (capacitor thermal noise, equal to the product of the Boltzmann constant, $k$, and the absolute temperature, $T$, divided by the capacitance, $C$ ) has no frequency component. This means that the voltage noise produced by a current discharged from a capacitor will scale linearly with time. As a result, one might expect to achieve a factor of $1 / \sqrt{t}$ in noise reduction by extending the charge accumulation time arbitrarily. This is not exactly how the ATONA functions, as one is able to subsample the measurement without losing the benefit of a longer integration time but the expected linear relationship is achieved, similar to previous systems in which the charge of the capacitor is read directly (Ireland et al., 2014). The theoretical noise floor of the ATONA design is not immediately apparent from the publicly available information about its capabilities, which does not reveal the design of the measurement circuit or the value of the capacitor employed. A simple calculation assuming kTC noise is the only source of noise on each ATONA measurement yields a value of $15-20 \mathrm{pF}$ for the complete circuit, which includes both the capacitor used on the amplifier and the capacitance of the Faraday collectors themselves as well as the wires and feedthroughs that connect them. We measure noise directly through a series of measurements on the Isotopx NGX with the instrument under vacuum, all lenses active, and the filament powered off. We then express this noise floor in terms of incident ion beam for direct comparison to RTIAs.

The results are shown in Table 1 and are plotted in two different ways. First, we show a series of measurements of ATONA noise compared to ideal RTIA J-N noise calculations for a series of RTIA resistor values in Fig. 3 (see Appendix A). This figure simply shows measurements taken with the ATONA with no ion beam, with the arithmetic mean of the measurements subtracted from each. This is, therefore, what a series of measurements of a stable beam would look like to the user during a measurement cycle. Each measurement is made with a $10 \mathrm{~s}$ integration, which is the typical integration time we use for the ATONA on most samples. The ATONA measurements have a standard deviation of $0.0085 \mathrm{fA}$, which is equivalent to $0.85 \mu \mathrm{V}$ on a $10^{11}$ RTIA. In Fig. 4, we show the same noise data as the $1 \sigma$ standard deviation of a signal plotted as a function of integration time to show the different behavior of the ATONA as integration time is changed. Using a $1 \mathrm{~s}$ or $100 \mathrm{~s}$ integration time, the ATONA measurements have standard deviations of 0.073 and $0.0018 \mathrm{fA}$, respectively. The $10 \mathrm{~s}$ integration time value compares favorably to a $10^{13}$ RTIA at $0.011 \mathrm{fA}$ but does not quite reach the low noise level of a $10^{14}$ RTIA at $0.0040 \mathrm{fA}$. 


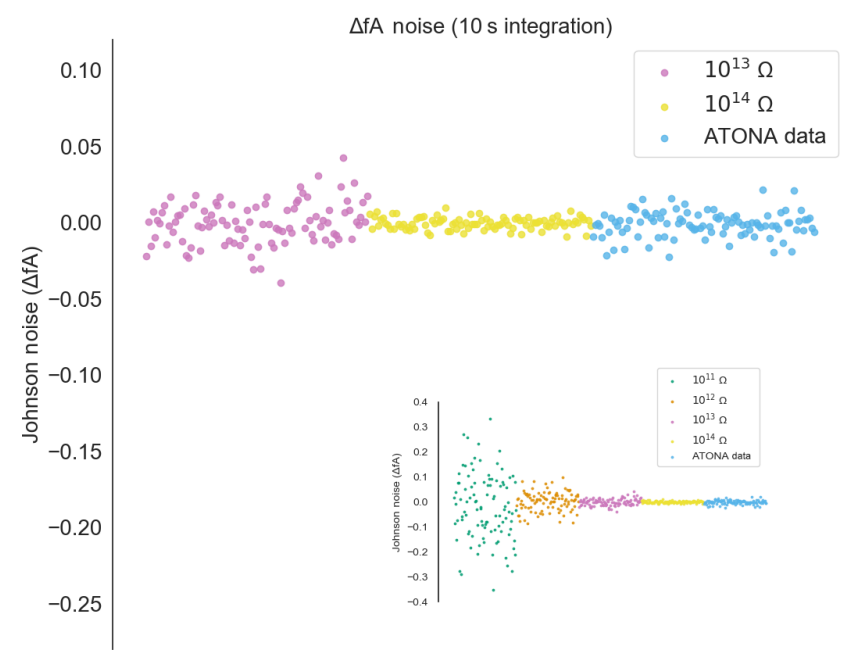

Figure 3. Measured noise on the ATONA amplifiers with $10 \mathrm{~s}$ integration periods expressed as deviations from the average signal with no ion beam in the mass spectrometer compared to ideal $10^{13}$ and $10^{14} \Omega$ RTIA noise. The signals are converted to equivalent ion beam current (see Sect. 3.1). Each measurement and ideal RTIA calculation is made over $10 \mathrm{~s}$ of integration and then simply plotted in order. The inset includes $10^{11}$ and $10^{12} \Omega$ examples as well, with the same ATONA data. Examples using 1 and $100 \mathrm{~s}$ integration times are included in the Appendix (Figs. A3 and A4), as is the full version of the inset (Fig. A5).

Similarly, at $1 \mathrm{~s}$ integration, the ATONA is between the $10^{12}$ and $10^{13}$ RTIA ( 0.40 and $0.037 \mathrm{fA}$, respectively).

\subsection{Air standards}

We prepared a large air standard of approximately $8.5 \times$ $10^{-13}$ mol of Ar per aliquot for mass spectrometer installation and initial testing. We used air taken at a distance from the Lamont-Doherty Earth Observatory Comer geochemistry building in Palisades, NY, on a dry day in November, and we filled the approximately $6 \mathrm{~L}$ standard tank with one aliquot from the approximately $0.1 \mathrm{cc}$ pipette. Subsequent aliquots for measurement were taken from the standard tank using the same pipette, attached to a custom-built high-vacuum system containing a hot SAES St101 getter. No primary volume calibration was performed on the pipette for the large standard, so the size of the Ar aliquot was first roughly estimated from the approximate volumes of the standard tank, pipette, and vacuum system, then calculated using intercalibration with a second standard tank with a manometrically calibrated pipette volume.

We measured four different splits of the air standard ranging from the full aliquot $\left(8.5 \times 10^{-13} \mathrm{~mol}^{40} \mathrm{Ar}\right)$ to approximately $0.36 \%$ of the total $\left(3.1 \times 10^{-15} \mathrm{~mol}^{40} \mathrm{Ar}\right)$. The split sizes of $100 \%, 17.7 \%\left(1.5 \times 10^{-13} \mathrm{~mol}^{40} \mathrm{Ar}\right)$, and $2.6 \%$ $\left(2.2 \times 10^{-14} \mathrm{~mol}^{40} \mathrm{Ar}\right)$ are most useful for comparing the Isotopx Xact RTIA to the ATONA. For all Xact measurements, a $10^{11} \Omega$ amplifier was used for ${ }^{40} \mathrm{Ar}$ and a $10^{12} \Omega$ ampli-

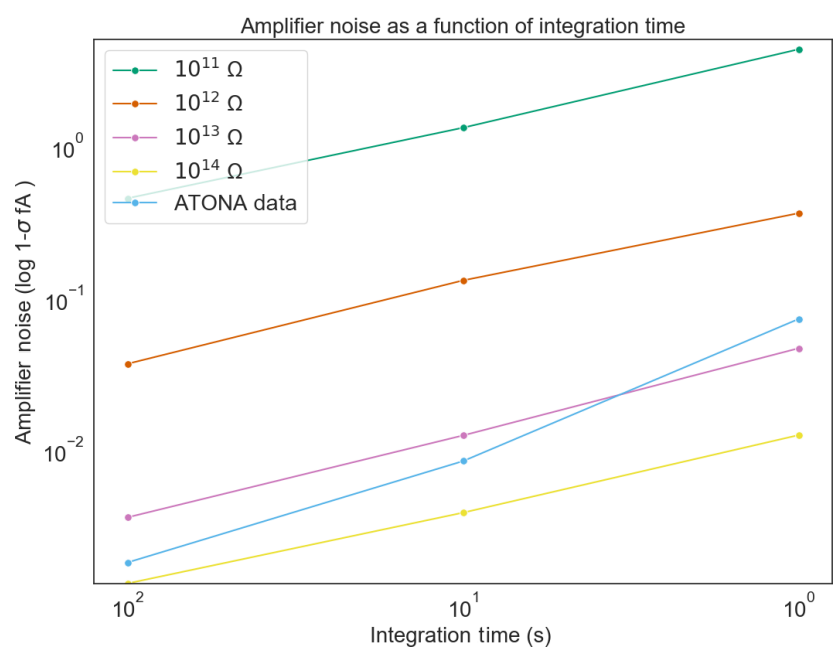

Figure 4. Noise expressed as the standard deviation of signal measured (ATONA) or calculated (ideal RTIA) for the ATONA and $10^{11}, 10^{12} \Omega, 10^{13}$, and $10^{14} \Omega$ RTIA. The ATONA noise decreases more quickly with increasing integration time because of the $1 / t$ (rather than $1 / \sqrt{t}$ ) relationship between noise and integration time.

fier was used for ${ }^{38} \mathrm{Ar}$. The ATONA amplifiers all use the same feedback capacitor and are therefore interchangeable. The ${ }^{40} \mathrm{Ar} /{ }^{38} \mathrm{Ar}$ ratios for these standards, which provide a direct comparison of the performance of the amplifiers without the effect of the ion-counting multiplier used to measure ${ }^{36} \mathrm{Ar}$, are shown in Fig. 5. For the different shot sizes, the Xact amplifiers produced standard deviations of $0.43 \%$, $3.07 \%$, and $27.9 \%$, while the ATONA amplifiers produced standards deviations of $0.21 \%, 1.35 \%$, and $7.87 \%$. As predicted based on zero-beam-noise measurements, the ATONA outperforms the Xact for all signal sizes. The improvement between the Xact and the ATONA is greater for smaller beam sizes because the effect of amplifier $\mathrm{J}-\mathrm{N}$ noise on the total uncertainty comes to dominate over other factors like source instability when the signal is smaller.

In order to provide a more rigorous assessment of the ATONA amplifiers themselves and to produce an amplifieronly dataset for ${ }^{40} \mathrm{Ar} /{ }^{36} \mathrm{Ar}$, which is a more commonly discussed isotope ratio in ${ }^{40} \mathrm{Ar} /{ }^{39} \mathrm{Ar}$ geochronology, we then switched to single-collector mode. Using the ATONA amplifiers, we measured each species by peak hopping on the $\mathrm{H} 2$ collector, which is normally used for ${ }^{40} \mathrm{Ar}$, and we measured ${ }^{40} \mathrm{Ar} /{ }^{38} \mathrm{Ar}$ and ${ }^{40} \mathrm{Ar} /{ }^{36} \mathrm{Ar}$ for splits of our air standard ranging from $200 \%$ (representing two aliquots of the full standard, $1.7 \times 10^{-12} \mathrm{~mol}$ of $\mathrm{Ar}$, or a beam of approximately $14400 \mathrm{fA}$ ) to $0.36 \%$, representing three splits with the extraction line, or approximately $3 \times 10^{-15} \mathrm{~mol}$ of $\mathrm{Ar}$ and a beam of $25.9 \mathrm{fA}$. The ${ }^{40} \mathrm{Ar} /{ }^{36} \mathrm{Ar}$ ratios for these measurements are shown in Fig. 6, and the ${ }^{40} \mathrm{Ar} /{ }^{38} \mathrm{Ar}$ ratios are shown in Fig. A1. The measured ratios along with internal uncertainties and standard deviations between analy- 
Table 1. Standard deviation of the background noise (unit: fA) for ideal RTIAs and actual standard deviation for measurements for the ATONA with no ion beam (unit: fA). A $1 \mathrm{fA}$ beam would produce $0.1 \mathrm{mV}$ on a $10^{11} \Omega$ RTIA.

\begin{tabular}{llllll}
\hline & $10^{11} \Omega$ RTIA & $10^{12} \Omega$ RTIA & $10^{13} \Omega$ RTIA & $10^{14} \Omega$ RTIA & ATONA \\
\hline $1 \mathrm{~s}$ & 0.4065498 & 0.12983724 & 0.04049524 & 0.01278319 & 0.07310124 \\
$10 \mathrm{~s}$ & 0.12743643 & 0.04051549 & 0.01269869 & 0.00408956 & 0.00850963 \\
$100 \mathrm{~s}$ & 0.04053762 & 0.01276568 & 0.00407741 & 0.00128429 & 0.00183472 \\
\hline
\end{tabular}

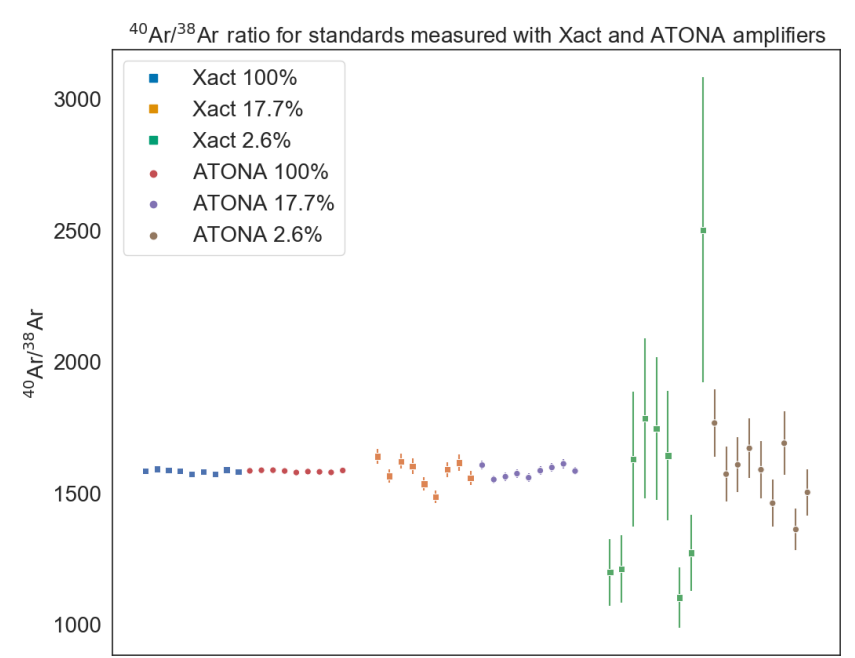

Figure $5 .{ }^{40} \mathrm{Ar} /{ }^{38} \mathrm{Ar}$ ratios for air standard splits of $100 \%, 17.7 \%$, and $2.6 \%$ measured using both the Isotopx Xact $\left(10^{11} \Omega\right.$ for ${ }^{40} \mathrm{Ar}$ and $10^{12} \Omega$ for ${ }^{38} \mathrm{Ar}$ ) amplifiers and the ATONA amplifiers in multicollection mode. Each sequence shows isotope ratios calculated from blank-corrected ratios of extrapolated peak heights for nine air standards measured sequentially, interspersed with blanks between each standard, for $600 \mathrm{~s}$ each. The ATONA measurements and the Xact measurements were both made using $6001 \mathrm{~s}$ integration periods; ATONA performance improves even further with longer integration periods.

ses are shown in Table 2 for ${ }^{40} \mathrm{Ar} /{ }^{36} \mathrm{Ar}$ and in Table A1 for ${ }^{40} \mathrm{Ar} /{ }^{38} \mathrm{Ar}$.

Finally, we measured the same ion beam $\left({ }^{40} \mathrm{Ar}\right)$ repeatedly on each Faraday detector to determine the gain bias between the different ATONA amplifiers. Choosing the axial detector as a reference, the relative gains of the other detectors ranged between $1.6 \%$ and $3.6 \%$ lower, with a standard deviation of between 106 and $220 \mathrm{ppm}$ for the intercalibration factor of each detector when measured using $1 \mathrm{~s}$ integration periods for 10 periods of $10 \mathrm{~s}$ on each detector (Fig. A2). Because we used a real Ar beam measured with a sequential peak hop rather than a synthetically produced calibration voltage, fluctuations in the ion source and mass analyzer electronics might also contribute noise to these measurements, so this is a maximum estimate of the intercalibration drift of the ATONA. The production model of the ATONA amplifiers, which are now being installed on some TIMS instruments, have a calibration voltage that eliminates these other sources

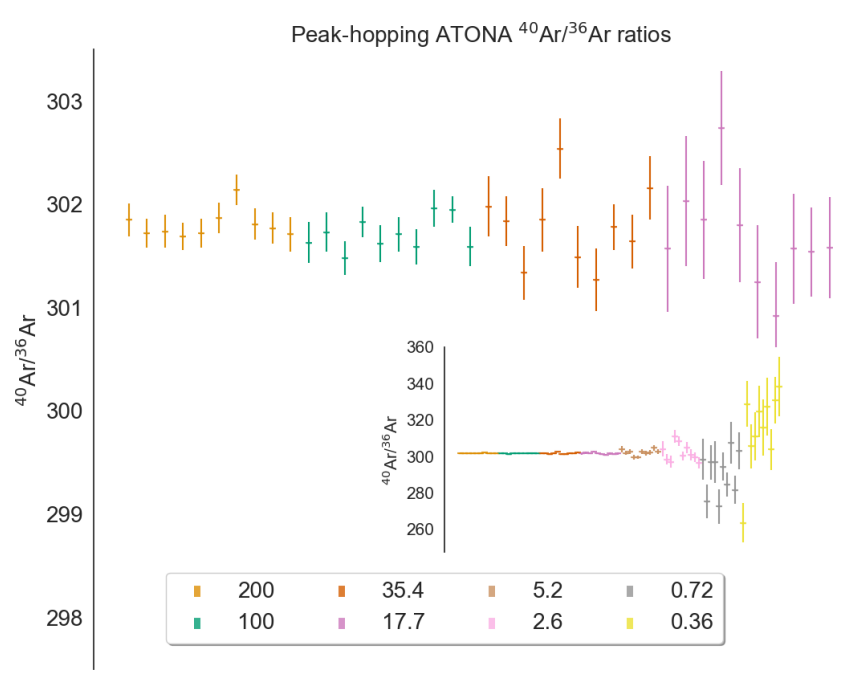

Figure 6. ${ }^{40} \mathrm{Ar} /{ }^{36} \mathrm{Ar}$ ratios for air standard splits from $200 \%$ to $17.7 \%$ (inset: $200 \%$ and $0.36 \%$ ) measured using the Isotopx ATONA amplifiers in single-collector peak-hopping mode, with the ${ }^{36} \mathrm{Ar},{ }^{38} \mathrm{Ar}$, and ${ }^{40} \mathrm{Ar}$ beams measured in sequence on the $\mathrm{H} 2$ Faraday. Each beam was measured in sets of three $10 \mathrm{~s}$ integration periods, which was repeated 10 times. Isotope ratios are calculated from blank-corrected ratios of extrapolated peak heights. Each sequence shows 10 air standards plotted interspersed for comparison.

of uncertainty; preliminary results from this system show a standard deviation of only $0.6 \mathrm{ppm}$ for each detector when measured using $2 \mathrm{~min}$ integration periods over multiple $4 \mathrm{~h}$ blocks (Szymanowski and Schoene, 2019).

Because the uncertainty of the measured signals is dominated by the thermal noise of the Faraday amplifier, the uncertainty of each measured ratio is controlled largely by the uncertainty of the smaller isotope. For comparison to other instruments, we plot each measured isotope ratio as a function of the sample size of the small isotope in the ratio in Fig. 7 (that is, for the same air standard, the ${ }^{40} \mathrm{Ar} /{ }^{36} \mathrm{Ar}$ ratio will plot approximately 5 times higher in terms of sample size than the ${ }^{40} \mathrm{Ar} /{ }^{38} \mathrm{Ar}$ ratio because the ${ }^{40} \mathrm{Ar} /{ }^{36} \mathrm{Ar}$ ratio of air is 298.56, while the ${ }^{40} \mathrm{Ar} /{ }^{38} \mathrm{Ar}$ ratio of air is 1583.87 ; Lee et al., 2006; Mark et al., 2011). This reference frame allows us to compare unlike detectors such as analog multipliers and Faraday cups, as well as to compare isotope ratios measured using a mix of detector types, such as the ${ }^{40} \mathrm{Ar} /{ }^{36} \mathrm{Ar}$ ratios measured in the standard multicollection mode of our NGX. 


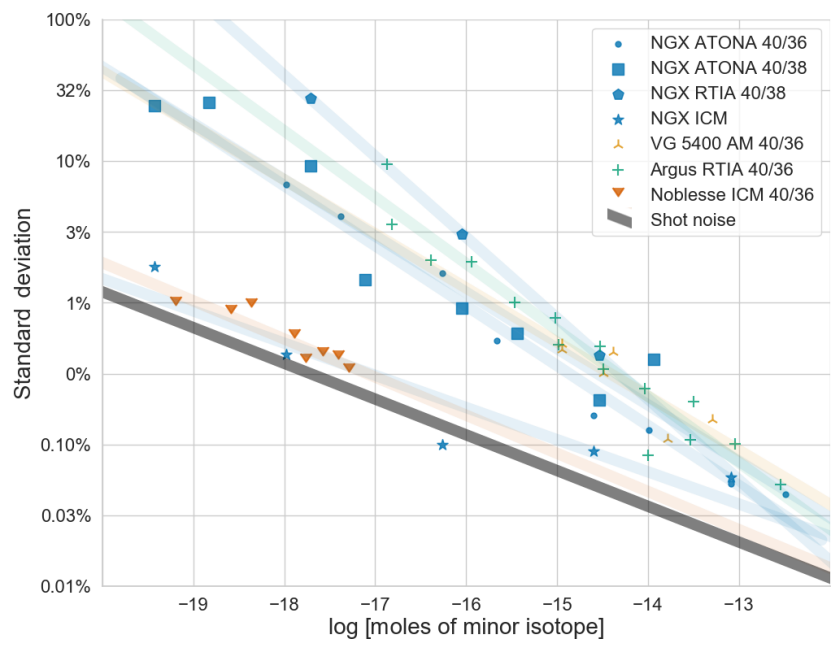

Figure 7. Standard deviation of measured ${ }^{40} \mathrm{Ar} /{ }^{36} \mathrm{Ar}$ or ${ }^{40} \mathrm{Ar} /{ }^{38} \mathrm{Ar}$ ratios for air standards measured on different mass spectrometers as a function of small isotope abundance in moles (see Sect. 3.2 for a description of data sources). Isotope ratios are calculated from blank-corrected ratios of extrapolated peak heights. The shaded lines are linear fits to each dataset, included primarily as a visual guide. ATONA is the ATONA amplifier described here, RTIA is a traditional resistor transimpedance Faraday amplifier, ICM is an ion-counting multiplier, and AM is an analog multiplier. The NGX data points with more than $10^{-17} \mathrm{~mol}$ of ${ }^{36} \mathrm{Ar}$ use an ATONA for the ${ }^{40} \mathrm{Ar}$ beam, but in all cases the uncertainty of the small isotope controls the uncertainty of the ratio. This plot provides a direct comparison of whole instrument performance rather than detector performance because the ion source and mass analyzer also contribute to uncertainty in the measurements, and the sample abundance is not weighted by source sensitivity. We note that we are not able to completely control for the effects of different analytical conditions, including background, detector integration time, total measurement time, sensitivity, and data reduction. The limit of shot noise, or counting noise, is shown in grey assuming no other sources of uncertainty and a regression through $600 \mathrm{~s}$ of analysis. The uncertainty of all detectors will approach this limit at large signals. Note that the uncertainty of the regression is approximately twice the uncertainty one would calculate from an average over the same interval in a mass spectrometry system without an evolving signal.

While a better reference frame for direct comparison of detector technologies might be beam size rather than sample size, the latter choice allows for a more realistic comparison of mass spectrometers as they are used in the laboratory. We also note that while most noble gas mass spectrometers provide a similar specification for constant-pressure ion source sensitivity, field reports indicate that some (notably the Thermo Argus) have an advantage due to both smaller volume and higher constant-pressure sensitivity. These results show a clear improvement for the NGX with ATONA compared to the previous generation of mass spectrometer (represented by the LDEO VG 5400) and the NGX with Xact $10^{12} \Omega$ RTIA (the same NGX at LDEO, with its original amplifiers). The performance is also better than published 
data for the Thermo Argus with $10^{12} \Omega$ RTIA (Mark et al., 2009) despite the Argus' apparently higher source sensitivity, which is consistent with the prediction that the ATONA will easily outperform a $10^{12} \Omega$ RTIA (Fig. 3); see Sect. 3.3 for a comparison to the Argus with a $10^{13} \Omega$ RTIA. Finally, the NGX using its ion-counting multiplier in peak-hopping mode is still able to achieve a much lower noise level for very small samples, comparable to the Nu Noblesse with multiple ioncounting multipliers (Jicha et al., 2016), which is also consistent with the predicted noise level of the ATONA. However, these detectors are limited to very small samples; the data points with more than $10^{-17} \mathrm{~mol}$ of ${ }^{36} \mathrm{Ar}$ in Fig. 7 actually use an ATONA for the ${ }^{40} \mathrm{Ar}$ beam, but we plot them in the ICM category because the uncertainty of the small isotope controls the uncertainty of the ratio measurement.

\subsection{APIS cocktail standards}

The Argon Intercalibration Pipette System (APIS) is a system designed to provide a portable set of argon gas standards of different size and isotope ratio for a noble gas mass spectrometer (Turrin et al., 2015). The APIS has three standard tanks containing air, a cocktail representing argon with an ${ }^{40} \mathrm{Ar} /{ }^{39} \mathrm{Ar}$ ratio typical of an irradiated Alder Creek sanidine standard, and a cocktail representing argon with an ${ }^{40} \mathrm{Ar} /{ }^{39} \mathrm{Ar}$ ratio typical of an irradiated Fish Canyon Tuff sanidine standard. Each tank has three pipettes attached to it, with volumes of $0.1,0.2$, and $0.4 \mathrm{cc}$, allowing aliquots of gas ranging in size from 1 to 7 times the size of the $0.1 \mathrm{cc}$ pipette to be extracted without resorting to multiple aliquots from a single pipette. We measured each possible size, 0.1 , $0.2,0.3,0.4,0.5,0.6$, and $0.7 \mathrm{cc}$, three times from each of the Alder Creek and Fish Canyon Tuff tanks and six times from the APIS air standard tank, interspersed with the lab air standard described earlier and procedural blanks.

The APIS standards have accumulated air background since the system was first deployed, so a direct comparison of measured ratios between labs is not possible. However, we can compare air-corrected values for the Fish Canyon and Alder Creek standard tanks - similar to what would be measured during an actual experiment. As an example, we plot measured radiogenic ${ }^{40} \mathrm{Ar} * /{ }^{39} \mathrm{Ar}$ values $\left({ }^{40} \mathrm{Ar} /{ }^{39} \mathrm{Ar}\right.$ ratios corrected for air contamination using simultaneously measured ${ }^{40} \mathrm{Ar} * /{ }^{36} \mathrm{Ar}$ ratios) for the Fish Canyon analog from the Isotopx NGX with the ATONA (10 s integration periods; $400 \mathrm{~s}$ measurement time) and the Thermo Argus with the $10^{12}$ and $10^{13} \Omega$ RTIA ( $1 \mathrm{~s}$ integration periods, $400 \mathrm{~s}$ measurement time; Fig. 8; Ross and Mcintosh, 2016). While the ATONA exhibits lower noise on a per-signal basis, the higher sensitivity of the Argus ion source makes the results indistinguishable.

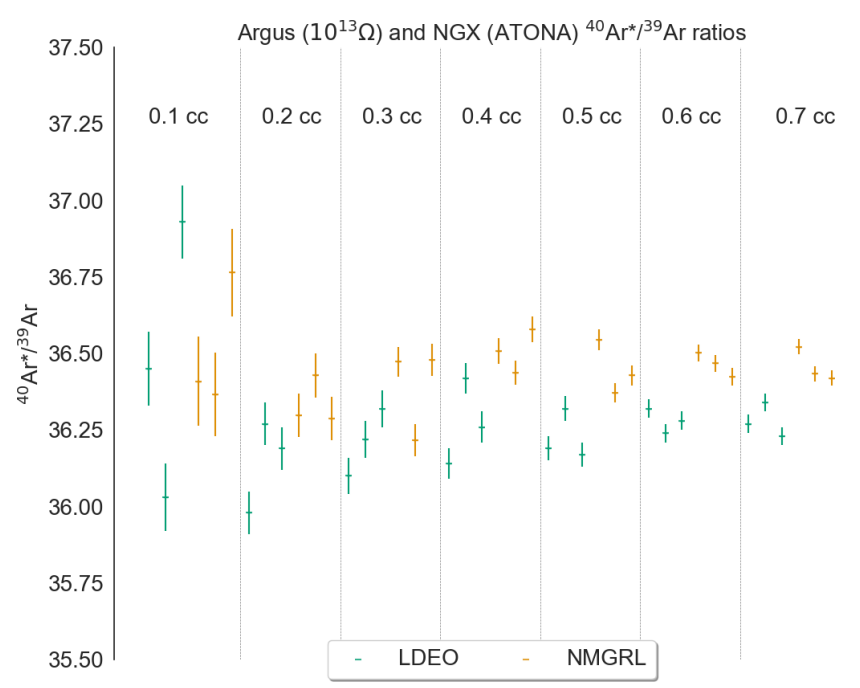

Figure 8. Air-corrected ${ }^{40} \mathrm{Ar} * /{ }^{39} \mathrm{Ar}$ ratios for the APIS Fish Canyon Tuff analog standard on the Isotopx NGX with the ATONA at Lamont-Doherty Earth Observatory and the Thermo Argus with the $10^{12}$ and $10^{13} \Omega$ RTIA at the New Mexico Geochronology Research Laboratory (Ross and Mcintosh, 2016), with smaller (0.1 cc) aliquots on the left and $0.2,0.3,0.4,0.5,0.6$, and $0.7 \mathrm{cc}$ aliquots to the right. Both sets of measurements were performed with $400 \mathrm{~s}$ of analysis time in multicollection. Isotope ratios are calculated from blank-corrected ratios of extrapolated peak height, with the ${ }^{40} \mathrm{Ar} *$ corrected for air contamination using the measured ${ }^{36} \mathrm{Ar}$. By design, the APIS experiments were conducted according to the same blank and standard protocols in each lab. The ATONA data were collected using $10 \mathrm{~s}$ integration periods, while the Argus data were collected using $1 \mathrm{~s}$ integration periods. The standard deviation of the signals for a given size aliquot is comparable for the two instruments.

\section{Summary}

The ATONA amplifier represents a significant step forward in Faraday cup amplifier technology for noble gas mass spectrometry. The ATONA allows a greater dynamic range of ion beams to be measured compared to existing RTIA technology, and only highly specialized RTIA electronics are able to compete with the low noise of the ATONA. The amplifiers are significantly more stable and have a higher dynamic range than ion-counting electron multipliers. Other types of mass spectrometers that produce a stable ion beam are likely to see an even greater performance improvement with the ATONA because of its ability to capitalize on long integration times to reduce noise. The strengths of the ATONA, combining low noise for small samples with a high dynamic range and good stability for large samples, are in harmony with the current priorities in the field of noble gas geochemistry, which require instruments that can deliver both high precision and flexibility for measuring a wide range of sample types. 


\section{Appendix A: Johnson-Nyquist noise calculation}

Thermal Johnson-Nyquist noise ( $\mathrm{J}-\mathrm{N}$ noise) is described by Eq. (4) from Nyquist (1928):

$V^{2}=4 R K_{\mathrm{B}} T$,

where $V$ is the voltage at the frequency of interest, $R$ is the resistance of the circuit, $K_{\mathrm{B}}$ is the Boltzmann constant, and $T$ is the temperature. We rearrange this to solve for voltage noise and then divide by the resistance of the circuit to arrive at the noise fluctuations in terms of beam current $I$.

$\sigma_{I}=\sqrt{4 R K_{\mathrm{B}} T} / R$

This equation is the basis for the calculations shown in Figs. 3, 4, A3, A4, A5, A6, and A7.

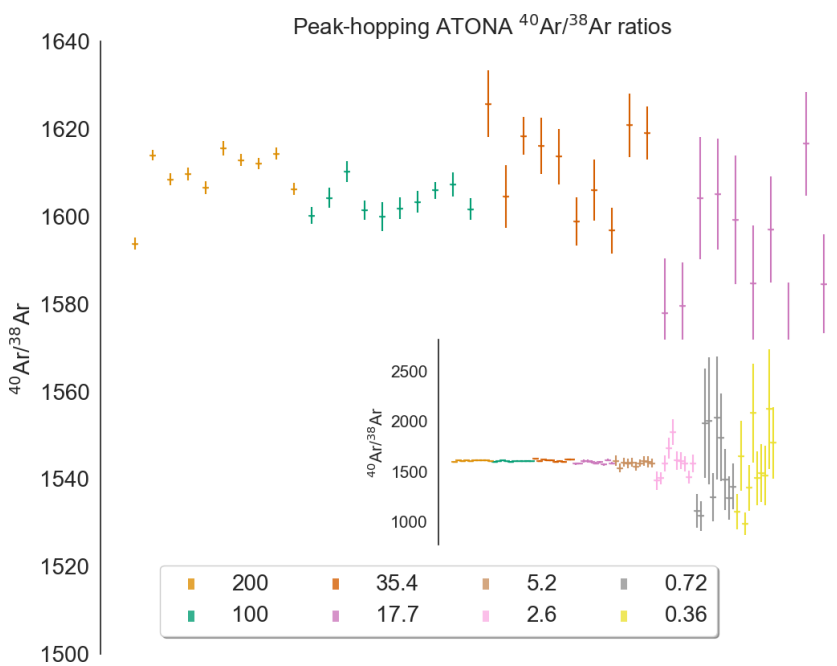

Figure A1. ${ }^{40} \mathrm{Ar} /{ }^{38} \mathrm{Ar}$ ratios for air standard splits from $200 \%$ to $17.7 \%$ (inset: $200 \%$ and $0.36 \%$ ) measured using the Isotopx ATONA amplifiers in single-collector peak-hopping mode, with the ${ }^{36} \mathrm{Ar},{ }^{38} \mathrm{Ar}$, and ${ }^{40} \mathrm{Ar}$ beams measured in sequence on the $\mathrm{H} 2$ Faraday. Each beam was measured in sets of three $10 \mathrm{~s}$ integration periods, which was repeated 10 times. Each sequence shows 10 air standards plotted interspersed for comparison.

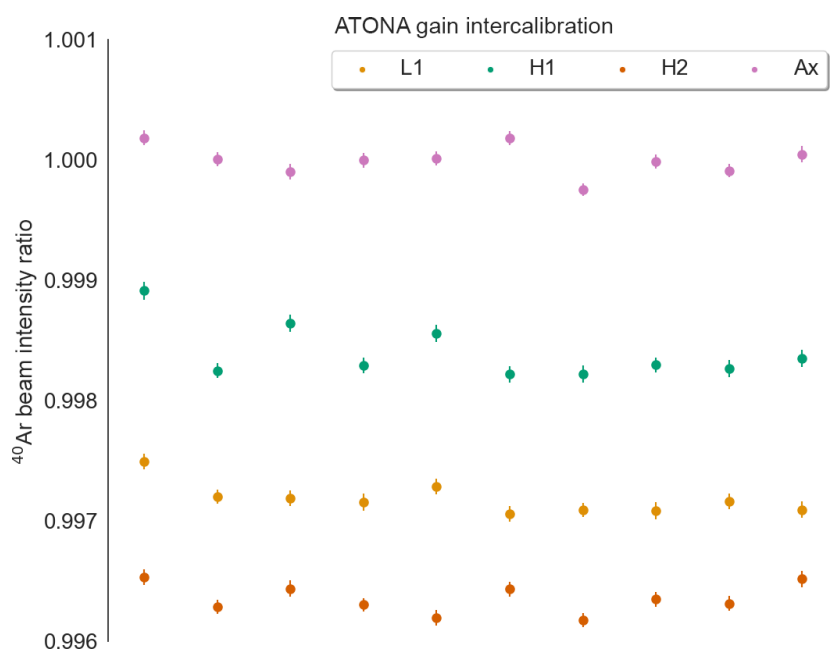

Figure A2. Intercalibration measurements using an ${ }^{40} \mathrm{Ar}$ beam produced by aliquots of the $8.5 \times 10^{-13} \mathrm{~mol}$ air standard, measured by peak hopping just the ${ }^{40} \mathrm{Ar}$ beam on each of the four ATONA Faraday collectors on the NGX. Plotted are the ratios of each measurement of the ${ }^{40} \mathrm{Ar}$ signal on a given detector to the average of all measurements on the axial detector. Measurements were made using $1 \mathrm{~s}$ integration periods in sets of 10 , repeated 10 times sequentially on each detector, with the intensities calculated using a linear extrapolation to time zero; internal uncertainties shown are the $1 \sigma$ standard error of the linear fit. No blank correction was made. The detector intercalibration factor ranges from 0.9964 to 0.9984 for the other three detectors relative to the axial detector, with standard deviations ranging from 106 to $220 \mathrm{ppm}$ for each.

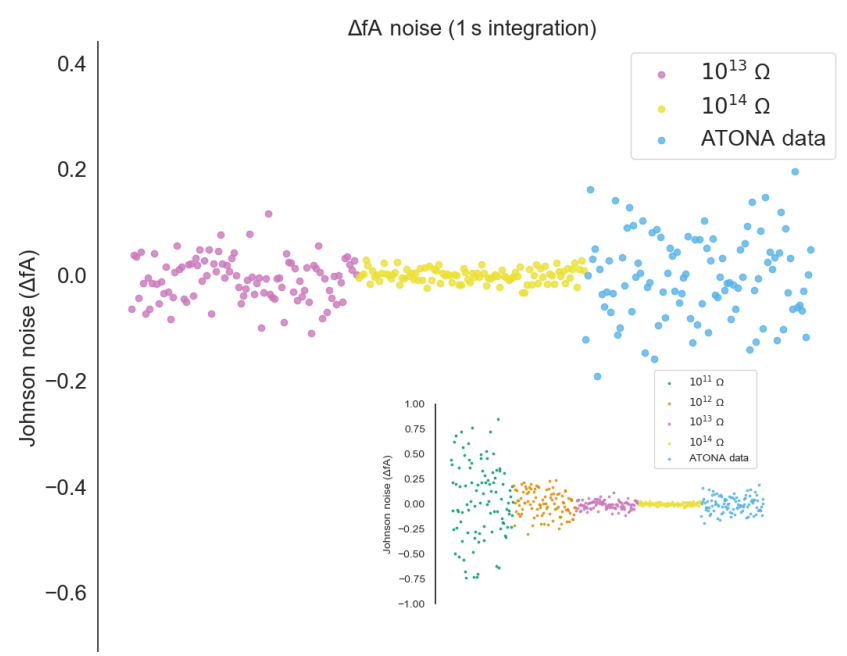

Figure A3. Measured noise on the ATONA amplifiers with $1 \mathrm{~s}$ integration periods expressed as deviations from the average signal with no ion beam in the mass spectrometer compared to ideal $10^{13}$ and $10^{14} \Omega$ RTIA noise. The signals are converted to equivalent ion beam current (see Sect. 3.1). Each measurement and ideal RTIA calculation is made over $1 \mathrm{~s}$ of integration and then simply plotted in order. The inset includes $10^{11}$ and $10^{12} \Omega$ examples as well, with the same ATONA data. Examples using a $10 \mathrm{~s}$ integration time are included in Fig. 3. The full version of the inset is provided in Fig. A6. 


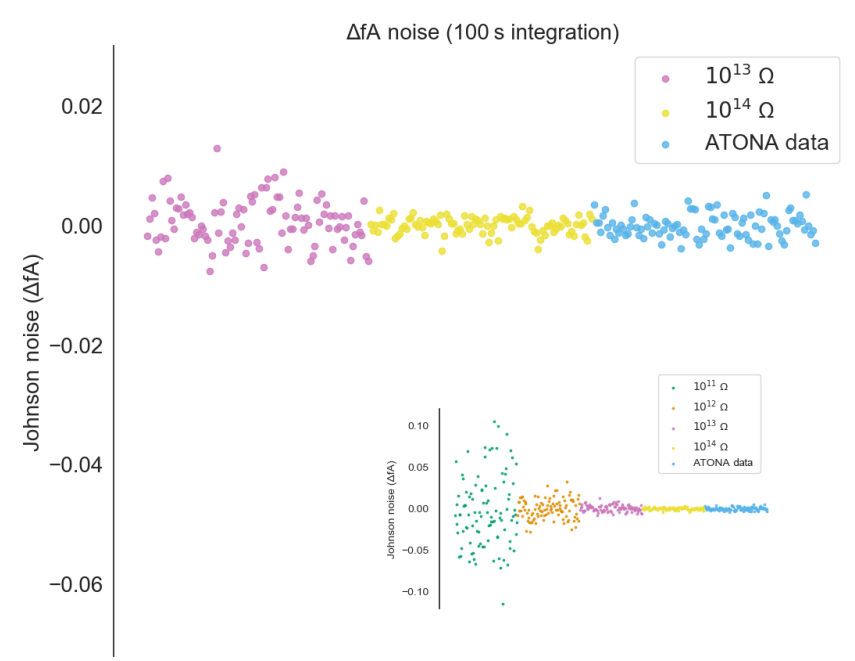

Figure A4. Measured noise on the ATONA amplifiers with $100 \mathrm{~s}$ integration periods expressed as deviations from the average signal with no ion beam in the mass spectrometer compared to ideal $10^{13}$ and $10^{14} \Omega$ RTIA noise. The signals are converted to equivalent ion beam current (see Sect. 3.1). Each measurement and ideal RTIA calculation is made over $100 \mathrm{~s}$ of integration and then simply plotted in order. The inset includes $10^{11}$ and $10^{12} \Omega$ examples as well, with the same ATONA data. Examples using a $10 \mathrm{~s}$ integration time are included in Fig. 3. The full version of the inset is provided in Fig. A7.

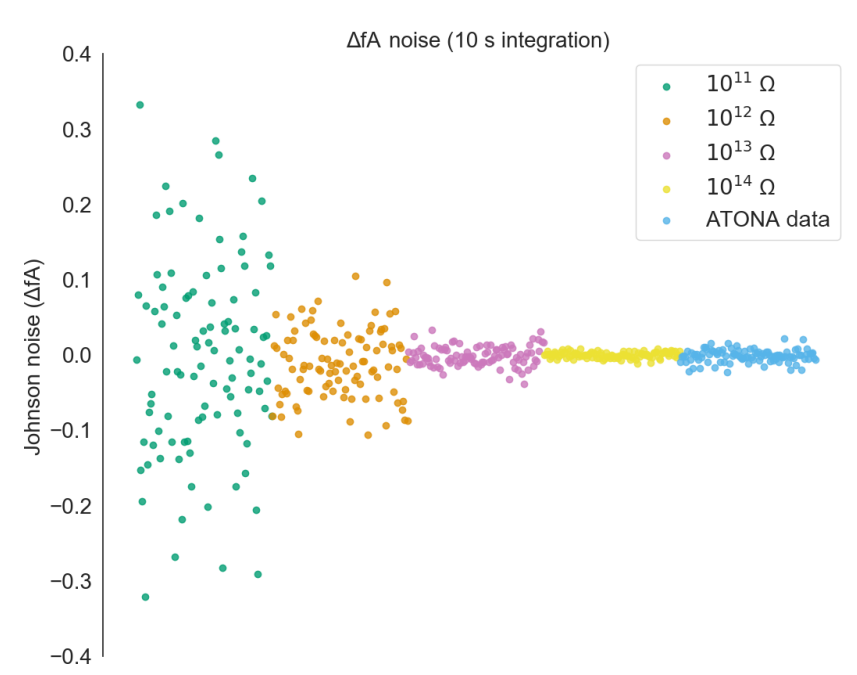

Figure A5. Measured noise on the ATONA amplifiers with $10 \mathrm{~s}$ integration periods expressed as deviations from the average signal with no ion beam in the mass spectrometer compared to ideal $10^{11}$, $10^{12}, 10^{13}$, and $10^{14} \Omega$ RTIA noise. The signals are converted to equivalent ion beam current (see Sect. 3.1). Each measurement and ideal RTIA calculation is made over $10 \mathrm{~s}$ of integration and then simply plotted in order. This is the full version of the inset from Fig. 3.

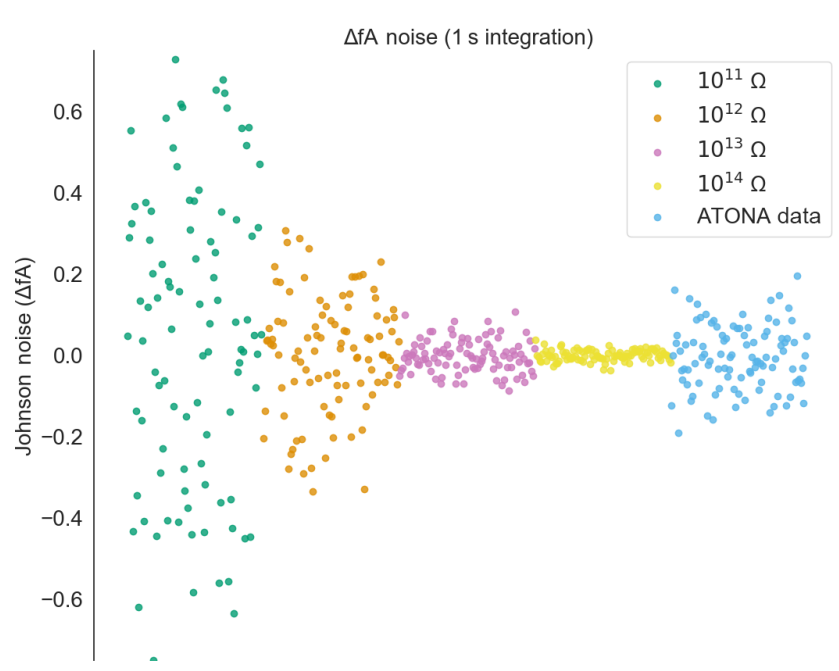

Figure A6. Measured noise on the ATONA amplifiers with $1 \mathrm{~s}$ integration periods expressed as deviations from the average signal with no ion beam in the mass spectrometer compared to ideal $10^{11}, 10^{12}$, $10^{13} \Omega$, and $10^{14} \Omega$ RTIA noise. The signals are converted to equivalent ion beam current (see Sect. 3.1). Each measurement and ideal RTIA calculation is made over $10 \mathrm{~s}$ of integration and then simply plotted in order. This is the full version of the inset from Fig. A3.

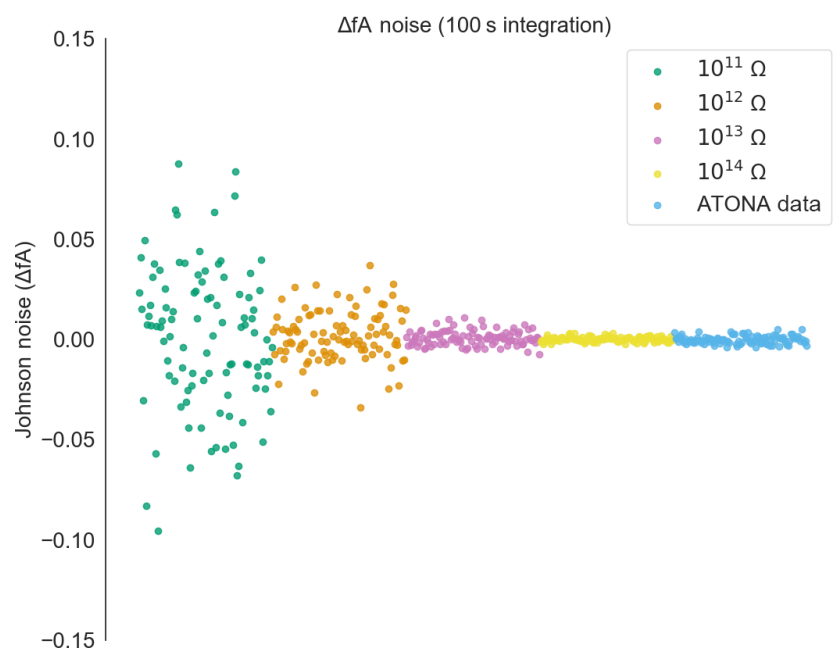

Figure A7. Measured noise on the ATONA amplifiers with $100 \mathrm{~s}$ integration periods expressed as deviations from the average signal with no ion beam in the mass spectrometer compared to ideal $10^{11}$, $10^{12} \Omega, 10^{13}$, and $10^{14} \Omega$ RTIA noise. The signals are converted to equivalent ion beam current (see Sect. 3.1). Each measurement and ideal RTIA calculation is made over $10 \mathrm{~s}$ of integration and then simply plotted in order. This is the full version of the inset from Fig. A4. 


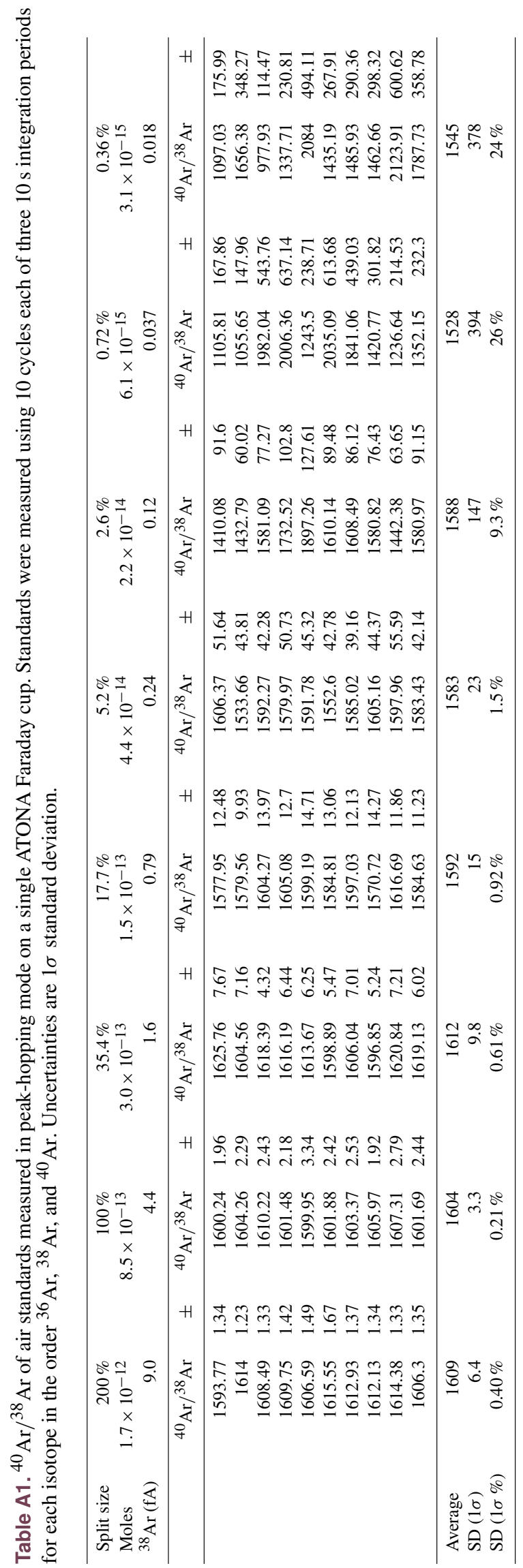


Data availability. Raw data collected at LDEO for this publication are archived at https://doi.org/10.5281/zenodo.3995136 (Cox, 2020).

Author contributions. SEC installed the instrument, set up hardware and software, designed experiments, performed measurements, and interpreted the results. SRH designed experiments, performed measurements, and interpreted results. DT designed and implemented the ATONA system and installed the prototype on the NGX at LDEO.

Competing interests. Damian Tootell is an employee of Isotopx, Ltd.

Acknowledgements. We thank Daniel Wielandt, Trevor Ireland, and Klaudia Kuiper for particularly thoughtful reviews that contributed greatly to the value of the paper. We thank Jake Ross for extensive discussion, Pychron programming help, and sharing raw data from NMGRL measurements published in conference proceedings as well as Brian Jicha for discussion and sharing raw data from WiscAr measurements published in Chemical Geology. We also thank Chris Varden for initial installation and testing of the NGX at LDEO.

Financial support. This research has been supported by the National Science Foundation, Directorate for Geosciences (grant no. 1636685).

Review statement. This paper was edited by Marissa Tremblay and reviewed by Daniel Wielandt and Trevor Ireland.

\section{References}

Bayer, R., Schlosser, P., Bönisch, G., Rupp, H., Zaucker, F., and Zimmek, G.: Performance and Blank Components of a Mass Spectrometric System for Routine Measurement of Helium Isotopes and Tritium by the $3 \mathrm{He}$ Ingrowth Method, Springer Berlin Heidelberg, Berlin, Heidelberg, https://doi.org/10.1007/978-3642-48373-8, 1989.

Burnard, P. G. and Farley, K. A.: Calibration of pressuredependent sensitivity and discrimination in Nier-type noble gas ion sources: TECHNICAL BRIEF, Geochem. Geophy. Geosy., 1, 2000GC000038, https://doi.org/10.1029/2000GC000038, 2000.

Coble, M. A., Grove, M., and Calvert, A. T.: Calibration of $\mathrm{Nu}$-Instruments Noblesse multicollector mass spectrometers for argon isotopic measurements using a newly developed reference gas, Chem. Geol., 290, 75-87, https://doi.org/10.1016/j.chemgeo.2011.09.003, 2011.

Cox, S. E.: Raw dataset for publication 10.5194/gchron-20201 [Data set], Zenodo, https://doi.org/10.5281/zenodo.3995136, 2020.
Esat, T. M.: Charge collection thermal ion mass spectrometry of thorium, Int. J. Mass Spectrom., 148, 159-170, https://doi.org/10.1016/0168-1176(95)04234-C, 1995.

Espanon, V. R., Honda, M., and Chivas, A. R.: Cosmogenic $3 \mathrm{He}$ and $21 \mathrm{Ne}$ surface exposure dating of young basalts from Southern Mendoza, Argentina, Quat. Geochronol., 19, 76-86, https://doi.org/10.1016/j.quageo.2013.09.002, 2014.

Ireland, T., Schram, N., Holden, P., Lanc, P., Ávila, J., Armstrong, R., Amelin, Y., Latimore, A., Corrigan, D., Clement, S., Foster, J., and Compston, W.: Charge-mode electrometer measurements of S-isotopic compositions on SHRIMP-SI, Int. J. Mass Spectrom., 359, 26-37, https://doi.org/10.1016/j.ijms.2013.12.020, 2014.

Jicha, B. R., Rhodes, J. M., Singer, B. S., and Garcia, M. O.: ${ }^{40} \mathrm{Ar} /{ }^{39} \mathrm{Ar}$ geochronology of submarine Mauna Loa volcano, Hawaii, J. Geophys. Res.-Sol. Ea., 117, B09204, https://doi.org/10.1029/2012JB009373, 2012.

Jicha, B. R., Singer, B. S., and Sobol, P.: Re-evaluation of the ages of ${ }^{40} \mathrm{Ar} /{ }^{39} \mathrm{Ar}$ sanidine standards and supereruptions in the western U.S. using a Noblesse multicollector mass spectrometer, Chem. Geol.y, 431, 54-66, https://doi.org/10.1016/j.chemgeo.2016.03.024, 2016.

Lee, J.-Y., Marti, K., Severinghaus, J. P., Kawamura, K., Yoo, H.S., Lee, J. B., and Kim, J. S.: A redetermination of the isotopic abundances of atmospheric Ar, Geochim. Cosmochim. Ac., 70, 4507-4512, https://doi.org/10.1016/j.gca.2006.06.1563, 2006.

Lu, Z.-T., Schlosser, P., Smethie, W., Sturchio, N., Fischer, T., Kennedy, B., Purtschert, R., Severinghaus, J., Solomon, D., Tanhua, T., and Yokochi, R.: Tracer applications of noble gas radionuclides in the geosciences, Earth-Sci. Rev., 138, 196-214, https://doi.org/10.1016/j.earscirev.2013.09.002, 2014.

Mark, D., Stuart, F., and de Podesta, M.: New high-precision measurements of the isotopic composition of atmospheric argon, Geochim. Cosmochim. Ac., 75, 7494-7501, https://doi.org/10.1016/j.gca.2011.09.042, 2011.

Mark, D. F., Barfod, D., Stuart, F. M., and Imlach, J.: The ARGUS multicollector noble gas mass spectrometer: Performance for ${ }^{40} \mathrm{Ar} /{ }^{39} \mathrm{Ar}$ geochronology, Geochem. Geophy. Geosy., 10, 2009GC002643, https://doi.org/10.1029/2009GC002643, 2009.

Mark, D. F., Renne, P. R., Dymock, R. C., Smith, V. C., Simon, J. I., Morgan, L. E., Staff, R. A., Ellis, B. S., and Pearce, N. J.: Highprecision ${ }^{40} \mathrm{Ar} /{ }^{39} \mathrm{Ar}$ dating of pleistocene tuffs and temporal anchoring of the Matuyama-Brunhes boundary, Quat. Geochronol., 39, 1-23, https://doi.org/10.1016/j.quageo.2017.01.002, 2017.

Nier, A. O.: A Mass Spectrometer for Routine Isotope Abundance Measurements, Rev. Sci. Instrum., 11, 212-216, https://doi.org/10.1063/1.1751688, 1940.

Nier, A. O.: A Mass Spectrometer for Isotope and Gas Analysis, Rev. Sci. Instrum., 18, 398-411, https://doi.org/10.1063/1.1740961, 1947.

Nyquist, H.: Thermal Agitation of Electric Charge in Conductors, Phys. Rev., 32, 110-113, https://doi.org/10.1103/PhysRev.32.110, 1928.

Renne, P. R., Swisher, C. C., Deino, A. L., Karner, D. B., Owens, T. L., and DePaolo, D. J.: Intercalibration of standards, absolute ages and uncertainties in ${ }^{40} \mathrm{Ar} /{ }^{39} \mathrm{Ar}$ dating, Chem. Geol., 145, 117-152, https://doi.org/10.1016/S0009-2541(97)00159-9, 1998. 
Reynolds, J. H.: High Sensitivity Mass Spectrometer for Noble Gas Analysis, Rev. Sci. Instrum., 27, 928-934, https://doi.org/10.1063/1.1715415, 1956.

Rose, J. and Koppers, A. A.: Simplifying Age Progressions within the Cook-Austral Islands using ARGUS-VI High-Resolution ${ }^{40} \mathrm{Ar} /{ }^{39} \mathrm{Ar}$ Incremental Heating Ages, Geochem. Geophy. Geosy., 20, 4756-4778, https://doi.org/10.1029/2019GC008302, 2019.

Ross, J. and Mcintosh, W.: Pychron: Everything you didn't know you wanted in a geochronology application [127-13], in: Geological Society of America Abstracts with Programs, vol. 48, The Geological Society of America, Denver, CO, https://doi.org/10.1130/abs/2016AM-286732, 2016.

Sprain, C. J., Renne, P. R., Wilson, G. P., and Clemens, W. A.: High-resolution chronostratigraphy of the terrestrial Cretaceous-Paleogene transition and recovery interval in the Hell Creek region, Montana, Geol. Soc. Am. Bull., 127, 393-409, https://doi.org/10.1130/B31076.1, 2015.

Szymanowski, D. and Schoene, B.: U-Pb TIMS Geochronology Using ATONA Amplifiers [V11D-0124], in: AGU Fall Meeting 2019, AGU, San Francisco, CA, 2019.
Turrin, B. D., Swisher, III, C. C., Hemming, S. R., Renne, P. R., Deino, A. L., Hodges, K. V., Van Soest, M. C., and Heizler, M. T.: An Update to the EARTHTIME Argon Intercalibration Pipette System (APIS): Smoking from the Same Pipe, in: AGU, Fall Meeting 2015, V53H-08, AGU, San Francisco, CA, available at: https://agu.confex.com/agu/fm15/meetingapp.cgi/Paper/ 84132 (last access: 21 August 2020), 2015.

Wijbrans, J., Schneider, B., Kuiper, K., Calvari, S., Branca, S., De Beni, E., Norini, G., Corsaro, R. A., and Miraglia, L.: ${ }^{40} \mathrm{Ar} /{ }^{39} \mathrm{Ar}$ geochronology of Holocene basalts; examples from Stromboli, Italy, Quat. Geochronol., 6, 223-232, https://doi.org/10.1016/j.quageo.2010.10.003, 2011.

Zhang, X., Honda, M., and Hamilton, D.: Performance of the High Resolution, Multi-collector Helix MC Plus Noble Gas Mass Spectrometer at the Australian National University, J. Am. Soc. Mass Spectr., 27, 1937-1943, https://doi.org/10.1007/s13361016-1480-3, 2016. 